wird durch das sich bildende Zinkhydrocarbonat milchig werden. Nachher bildet sich allmählich im Innern eine Schicht derselben Verbindung, die an dem Metall festhaftet und es vor der weiteren Berührung mit dem Wasser schützt. Ebenso verhält es sich mit Bleiröhren, die mit Wasser Bleihydrocarbonat bilden.

A rsen: Sanitäre Bestimmungen haben arsenhaltige Farben für Tapetèn und Gegenstände häuslichen Gebrauchs verboten. Arsen findet sich aber in Nahrungs- und Genußmitteln, im Wein, Bier, in der Weinsäure, in komprimierter Kohlensäure, im Glycerin, in der Schwefelsäure, in gewissen Entfärbungskohlen, in Citronensäure u. s.w. So fand $i c h^{1}$ ) in einem Wein einmal $0,135 \mathrm{~g}$ Arsenigsäureanhydrid im Liter, zum Glücke konnte dieser Wein sogleich mit Beschlag belegt werden, bevor er noch tödliche Vergiftungen bewirkt haben konnte.

Man trifft heute Wein an, der aus einem anderen Grunde Arsen enthalten kann, wenn nämlich arsenhaltige Mittel zur Bekämpfung schädlicher Insekten beim Weinstock verwendet worden sind. So benutzt man für diesen Zweck bekanntlich Bleiarseniate, Alkaliarsenite und-arseniate, Kupferarseniat u. s. w. Nach Erfahrungen in Frankreich kann ein Wein von derartig behandelten Weinstöcken Arsen enthalten. Die französische Regierung hat auf Grund zahlreicher Analysen und Gutachten verschiedener Forscher am 23. Oktober 1908 die Anwendung derartiger Arseniate zur Bekämpfung von Insekten bei Weinstöcken verboten, auch im Hinblick auf Vergiftungen, die beim Gebrauch der Arseniate bei den sie verwendenden Landleuten eintreten können.

${ }^{3)}$ Rev, intern. des falsifications 1906, 19, 84-86.

(Aus dem Italienischen ubersetzt von W. Roth.)

\title{
Die Bestimmung des Stärkesirups in Pflaumenmarmeladen.
} Von

\section{A. Beythien.}

Mitteilung aus dem Chemischen Untersuchungsamte der Stadt Dresden. [Eingegangen am 18. Dezember 1910]

Mehrere von Fachgenossen und Fabrikanten an mich gerichtete Anfragen deuten darauf hin, daß bezüglich der Beurteilung von Zwetschen- und Pflaumenmarmeladen die Ansicht herrscht, man müsse vor der Berechnung des Stärkesirupgehaltes von der ermittelten spezifischen Drehung der invertierten Trockensubstanz den Wert 21,5 subtrahieren und erst die der Differenz entsprechende Zahl aus der Juckenack'schen Tabelle entnehmen. Offenbar ist diese Ansicht durch die Tatsache hervorgerufen, daß Jucken $\mathrm{ack}^{1}$ ) bei mehreren Proben Pflaumenmus die spezifische Drehung der invertierten Trockensubstanz zu ungefähr 0 fand und selbst in ähnlicher Weise den Stärkesirupgehalt mehrerer verfälschter Pflaumenmuse berechnete $^{2}$ ). Eine einfache Überlegung lehrt aber, daß diese Art der Berechnung zu unrichtigen Werten führen muß.

1) Diese Zeitschrift 1904, 8, 32.

2) Daselbst.S. 33, Fußnote. 
Die spezifische Drehung eines Gemisches aus 2 Stoffen von verschiedenem Drehungsvermögen ist eine Funktion der spezifischen Drehung ihrer Einzelbestandteile, und man kann daher sowohl aus dem Prozentgehalte der Komponenten die spezifische Drehung der Mischung, als auch umgekehrt aus der spezifischen Drehung den Gehalt an den Bestandteilen ableiten.

Die einfachste geometrische Darstellung dieser Gesetzmäßigkeit stellt, wie ich in meiner Abhandlung „Beiträge zur quantitativen Analyse organischer Gemische mit Hilfe des Refraktometers"1) ausgeführt habe, die gerade Linie dar, deren Endpunkte man erhält, wenn man die spezifische Drehung der Einzelbestandteile als Ordinaten in ein rechtwinkliges Koordinatensystem cinzeichnet und als zugehörige Abszisse für den schwächer drehenden Anteil den Nullpunkt des Systems, für den stärker drehenden hingegen den Punkt 100 wählt.

In der Gleichung der geraden Iinie:

$$
\begin{aligned}
& y=a x+b ; \\
& x=\frac{y-b}{a}
\end{aligned}
$$

bedeutet hier die Ordinate $\mathrm{y}$ die spezifische Drehung und die Abszisse $\mathbf{x}$ den Prozentgehalt der Mischung an dem stärker drebenden Anteil. a ist die Tangente des Winkels $(\alpha)$ zwischen der Geraden und der Abszissen-Achse, b der Abstand zwischen dem Schnittpunkte der Geraden mit der Ordinaten-Achse und dem Nullpunkte des Systems. Bezeichnet man die spezifische Drehung des schwächer drehenden Bestandteils mit $d_{2}$ und diejenige des stärker drehenden mit $d_{1}$, so nehmen die Gleichungen nach der früher gegebenen Ableitung folgende Form an:

$$
\begin{aligned}
& y=\frac{d_{1}-d_{2}}{100} \cdot x+d_{2} \\
& x=\frac{100}{d_{1}-d_{2}}\left(y-d_{2}\right) .
\end{aligned}
$$

Habe ich also die spezifische Drehung einer Mischung (D) bestimmt, so brauche ich den Wert $D$ nur für $y$ einzusetzen und erhalte den Prozentgehalt des stärker drehenden Bestandteils zu:

$$
\mathrm{x}=\frac{100}{\mathrm{~d}_{1}-\mathrm{d}_{2}}\left(\mathrm{D}-\mathrm{d}_{2}\right)
$$

Bei der Ableitung seiner Tabelle ging nun Juckenack von der Annahme aus, daß die invertierte Trockensubstanz der meisten Früchte die gleiche spezifische Drehung wie der Invertzucker, nämlich $-21, \tilde{5}$, besitzt, während er für den Stärkesirup eine spezifische Drehung von $+134,1$ zugrunde legt. Unter dieser Voraussetzung ist also für $d$ der Wert $+134,1$ und für $d_{2}$ der Wert $-21,5$ einzusetzen, und die Gleichung für $x$ nimmt folgende Form an:

$$
x=\frac{100}{134,1+21,5}(D+21,5)=\frac{100}{155,6}(D+21,5) \text {. }
$$

Für reinen Stärkesirup wird $\mathrm{D}=+134,1$ und $\mathrm{x}=100$; für reinen Invertzucker wird $\mathrm{D}=-21,5$ und $\mathrm{x}=0$.

Die Gerade zeigt daun folgenden Verlauf (Linie I):

1) A. Beythien und R. Hennicke, Pharm. Zentralhalle 1907, 48, 1005. 
Ein Gemiseh von Pflaumenmus mit der spezifischen Drehung \pm 0 und ron Stärkesirup mit der spezifischen Drehung $+134,1$ folgt der gleichen Gesetzmäßigkeit wie das oben gewählte Beispiel. Wenn ich aber die Werte für $\mathrm{x}$ in der Weise berechnen wollte, daß ich von der beobachteten spezifischen Drehung D einfach die Zahl 21,5 subtrahierte und im übrigen die Juckenack'sche Tabelle, also die oben für Linie I abgeleitete Gleichung benutzte, so würde ich zu folgendem Ergebnis gelangen :

$$
\begin{aligned}
& x=\frac{100}{134,1+21,5}(D-21,5+21,5) \\
& y=a x .
\end{aligned}
$$

Das ist die Gleichung der durch den Nullpunkt zu Linie I gelegten Parallelen (Linie II). Daß sie keinen Ausdruck für den Gehalt an Stärkesirup darbietet, ergibt sich ohne weiteres, wenn man vom reinen Stärkesirup ausgehend D $=+134,1$ seizt. Dann wird $x=\frac{100}{155,6} \cdot 134,1$, das heißt reiner Stärkesirup enthält nur $\frac{134,1}{155,6} \times 100$

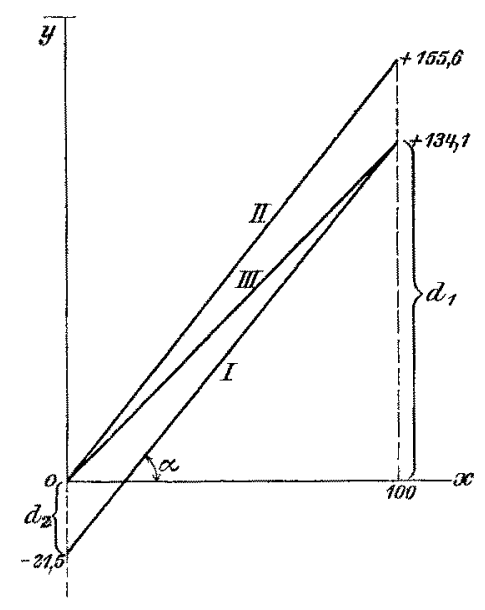
$=86,1 \%$ Stärkesirup.

Die richtige Linie erhält man selbstredend durch Verbindung des Nullpunktes mit der Ordinate 134,1 (Linie III), und ihre Gleichung ist:

$$
\begin{aligned}
& y=\frac{134,1}{100} \cdot x \\
& x=\frac{100}{134,1} \cdot D .
\end{aligned}
$$

Ein praktisches Beispiel möge vorstehenden Gedankengang noch etwas anschaulicher gestalten:

Angenommen, ich vermische:

100 Teile Pflaumenmus, dessen Gehalt an wasserlöslichem Extrakt $66,9 \%$ beträgt, mit

100 Teilen Stärkesirup (Wassergehalt $18 \%$, Extrakt $82 \%$ ), so erhalte ich ein Produkt, dessen Gehalt an Stärkesirup $50 \%$ beträgt.

Unter der Voraussetzung, daß die spezifische Drehung des invertierten Extraktes beim Stärkesirup $d_{1}=+134,1$ und beim Pflaumenmus $d_{2}= \pm 0$ beträgt, kann ich die spezifis che Drehung des Gemisches $\mathrm{D}$ in folgender Weise berechnen:

d. h.

$$
\mathrm{d}_{1}=+134,1
$$

100 Teile Extrakt aus Stärkesirup, zu $100 \mathrm{ccm}$ gelöst, drehen im $100 \mathrm{~mm}$-Rohr $+134,1^{0}$

$82 " \quad " \quad$ " " $" 100 "$ " " $" 100 " \quad+109,96^{\circ}$

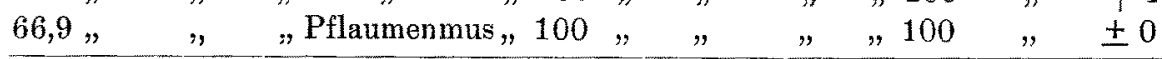

148,9 Teile Extrakt der Mischung, zu $100 \mathrm{ccm}$ gelöst, drehen im $100 \mathrm{~mm}$-Rohr $+109,96^{\circ}$.

Die spezifische Drehung des invertierten Extraktes der Mischung ist also

$$
\mathrm{D}=\frac{109,96 \times 100}{148,9}=+73,85^{\circ} \text {. }
$$

N. 11. 
Aus D berechnet man nach den vorstehend besprochenen Methoden den Gehalt an Stärkesirup:

a) Nach der Juckenack'schen oder nach der von mir und Simmich mitgeteilten Tabelle entspricht der spezifischen Drehung $\mathrm{D}=+73,85$ ein Gehalt des invertierten Extraktes an wasserhaltigem Stärkesirup von $74,85 \%$

$100 \mathrm{~g}$ Extrakt enthalten . . . . . . . 74,85 g

$74,45 \mathrm{~g} "$ oder $100 \mathrm{~g}$ der Mischung enthalten $74,85 \times 0,7445 \mathrm{~g}$ $=55,73 \%$ Stärkesirup.

Das Ergebnis ist um 5,73\% zu hoch:

b) Subtrahiert man von der spezifischen Drehung den Wert 21,5, so entspricht der Differenz $73,85-21,50=52,35$ nach der Juckenack'schen Tabelle ein Stärkesirupgelalt (wasserhaltig) von $57,88 \%$.

$100 \mathrm{~g}$ Extrakl enthalten . . . . . . . $57,88 \mathrm{~g}$

$74,45 \mathrm{~g} \quad " \quad$ oder $100 \mathrm{~g}$ der Mischung enthalten $57,88 \times 0,7445 \mathrm{~g}$ $=43,09 \%$ Stärkesirup.

Das Ergebnis ist um 6,91\% zu niedrig!

c) Nach der Gleichung $x=\frac{100}{134,1} \times D$ ergibt sich der Gehalt des Extraktes an wasserfreiem Stärkesirup zu $\frac{100}{134,1} \times 73,85=55,07 \%$

$100 \mathrm{~g}$ Extrakt enthalten . . . . . . $55,07 \mathrm{~g}$

$74,45 \mathrm{~g}, \quad$ oder $100 \mathrm{~g}$ der Mischung enthalten $55,07 \times 0,7445$

$=41 \%$ wasserfreien oder $50 \%$ wasserhaltigen Stärkesirup.

Das Ergebnis ist richtig!

Bei der Beurteilung von Pflaumen-Marmeladen ist nun zu berücksichtigen, daß diese weit weniger Obstbestandteile enthalten als das eben besprochene Gemisch von Pflaumenmus und Stärkesirup. Bei diesen tritt also die spezifische Drehung des Pflaumenextraktes hinter derjenigen des Stärkesirups zurück, und das Juckenack'sche Verfahren wird daher der Wahrheit näher kommende, das unter b) angeführte aber noch stärker abweichende Werte ergeben.

Auch diese Tatsache möge durch ein praktisches Beispiel näher illustriert werden.

Angenommen, ich verkoche 100 Teile frische Pflaumen (Zwetschen) und 100 Teile Stärkesirup zu Marmelade, deren Wassergehalt $25 \%$ beträgt.

Die Pflaumen mögen nach der Analyse 4 von E. Hotter ${ }^{1}$ ) 83,17\% Wasser, 16,83\% Trockensubstanz und 16,22\% löslichen Extrakt enthalten. Die spezifische Drehung des invertierten Pflaumenextraktes sei \pm 0 .

Die Zusammensetzung der fertigen Marmelade ergibt sich aus folgendem Ansatz:

100 Teile Pflaumen mit $16,83 \mathrm{~g}$ Trockensubstanz und

100 Teile Stärkesirup mit $82,00 \mathrm{~g}$

im ganzen 200 Teile Mischung mit $98,83 \mathrm{~g}$ Trockensubstanz ergeben x Teile Marmelade mit $75 \%$ oder mit $\frac{75 \times \mathrm{x}}{100} \mathrm{~g}$ Trockensubstan $z$

$$
\begin{aligned}
\frac{75 \mathrm{x}}{100} & =98,83 \\
\mathrm{x} & =131,8 \mathrm{~g} .
\end{aligned}
$$

1) Mitteilung der Landw.chem. Versuchsstation Graz 1906, 38. 
Um eine Marmelade von $25 \%$ Wassergebalt zu erzielen, muß man also die $200 \mathrm{~g}$ Gemisch bis auf $131,8 \mathrm{~g}$ einkochen.

$131,8 \mathrm{~g}$ Marmelade enthalten dann $100 \mathrm{~g}$ wasserhaltigen Stärkesirup mit $82 \mathrm{~g}$ Extrakt und 16,22 g Extrakt aus Pflaumen;

$100 \mathrm{~g}$ Marmelade enthalten also $75,87 \mathrm{~g}$ wasserbaltigen Stärkesirup mit 62,21 g Extrakt und 12,31 g Extrakt aus Pflaumen, im Ganzen also $74,52 \mathrm{~g}$ Extrakt.

Die spezifische Drehung des Marmeladenextraktes D berechne ich wie bei dem vorigen Beispiel:

100 Teile Extrakt aus Stärkesirup, zu $100 \mathrm{~cm}$ gelöst, drehen $+134,1^{0}$

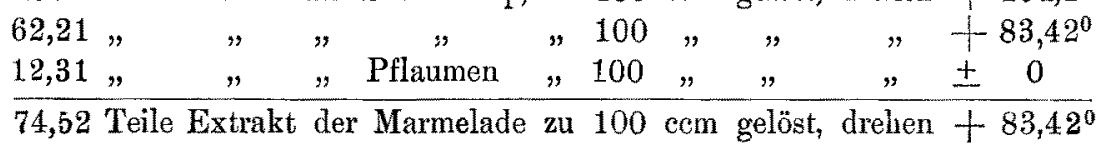

$100 " \quad " \quad " \quad " 100, \quad " \quad, \quad " \quad+\frac{83,42 \times 100}{74,52}$

Berechnet man aus D wieder nach den drei angeführten Methoden den Gehalt an Stärkesirup, so ergibt sich folgendes Bild:

a) Nach der Tabelle von Juckenack entspricht der spezifischen Drehung $+111,94^{0}$ ein Gehalt des invertierten Extraktes von $104,55 \%$ wasserhaltigem Stärkesirup.

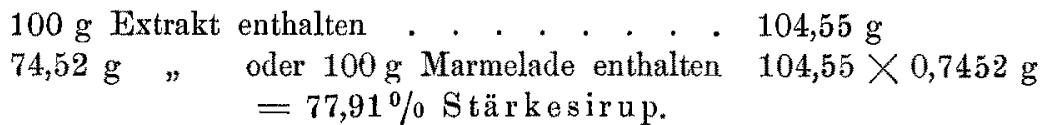
zu hoch!

Der tatsächliche Gehalt ist $75,87 \%$ und das Resultat ist sonach un $2,04 \%$

b) Subtrahiert man von der spezifischen Drehung den Wert 21,5 , so entwpricht der Differenz 111,94-21,5 = 90,44 nach der Juekenack'schen Tabelle ein Gehalt von $87,75 \%$ wasserhaltigem Stärkesirup, d. h.

$100 \mathrm{~g}$ Extrakt enthalten $\quad$ oder $100 \mathrm{~g}$ Marmelade enthalten $87,75 \times 0,7452 \mathrm{~g}$
$74,52 \mathrm{~g} \quad=65,39 \%$ Stärkesirup.

Das Ergebnis ist um 10,52\% zu niedrig!

c) Nach der Gleichung $\mathrm{x}=\frac{100}{134,1} \times \mathrm{D}$ ergibt sich der Gehalt des Extraktes an wasserfreiem Stärkesirup zu $\frac{100}{134,1} \times 111,94=83,48 \%$.

$100 \mathrm{~g}$ Extrakt enthalten . . . . . . . $83,48 \mathrm{~g}$

$74,52 \mathrm{~g} \# \quad$ oder $100 \mathrm{~g}$ Marmelade enthalten $83,48 \times 0,7452 \mathrm{~g}$

$=62,21 \%$ wasserfreien oder $75,87 \%$ wasserhaltigen Stärkesirup.

Das Ergebnis ist richtig!

Für Marmeladen aus Frucht, Zucker und Stärkesirup läßt sich nur dann eine exakte Gleichung aufstellen, wenn die spezifische Drehung des invertierten Obstextraktes gleich derjenigen des Invertzuckers, nämlich gleich $-21,5^{0}$ ist. Sobald aber die spezifische Drehung des Obstextraktes einen anderen Wert annimmt, entfällt diese Möglichkeit, weil zurzeit kein Mittel besteht, den Obstgehalt zu bestimmen, und man muß sich daher in solchen Fällen mit einer annähernden Bestimmung begnügen. 
Daß hierzu, auch für Pflaumenmarmelade, die vereinbarte Methode nach Juckenack völlig ansreicht, steht schon auf Grund des vorigen Beispiels zweifellos fest. Durch eine nochmalige Rechnung möge aber veranschaulicht werden, daß bei Gegenwart von Invertzucker auch nach der vereinbarten Methode fast absolut richtige Werte erhalten werden.

Angenommen ich verkoche 100 Teile frische $\mathrm{Zwetschen}$ mit $16,83 \%$ Trockensubstanz und 16,22\% löslichem Extrakt, fermer 50 Teile Invertzucker und 50 Teile Stärkesirup zu einer Marmelade, deren Wassergehalt $25 \%$ beträgt, so ergibt sich der Grad der Einkochung und die Zusammensetzung der fertigen Marmelade nach folgendem Ansaize:

100 Teile Zwetschen mit $16,83 \mathrm{~g}$ Trockensubstanz

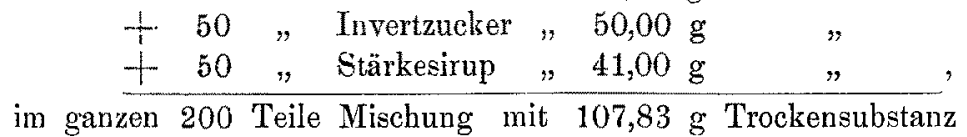

ergeben $\mathrm{x}$ Teile Marmelade mit $75 \%$ oder $\frac{75 \mathrm{x}}{100} \mathrm{~g}$ Trockensubstanz.

$$
\begin{aligned}
\frac{75 \mathrm{x}}{100} & =107,83 \\
\mathrm{x} & =143,77 \mathrm{~g} .
\end{aligned}
$$

Um eine Marmelade von $25 \%$ Wassergehalt zu erzielen, muß man also die $200 \mathrm{~g}$ Mischung bis auf $143,77 \mathrm{~g}$ einkochen.

$143,77 \mathrm{~g}$ Marmelade enthalten dann $50 \mathrm{~g}$ Invertzucker, $50 \mathrm{~g}$ wasserhaltigen Stärkesirup mit $41 \mathrm{~g}$ Extrakt und $16,22 \mathrm{~g}$ Extrakt aus Pflaumen.

$100 \mathrm{~g}$ Marmelade enthalten also $34,77 \mathrm{~g}$ Invertzucker, $34,77 \mathrm{~g}$ wasserhaltigen Stärkesirup mit 28,53 g Extrakt und 11,28 g Extrakt aus Pflaumen, in Summa $74,58 \mathrm{~g} \mathrm{Extrakt.}$

Die spezifische Drehung des invertierten Marmeladenextraktes D berechnet sich unter der Annahme, daß die spezifische Drehung des Pflaumenextraktes \pm 0 sei, in folgender Weise:

$\mathrm{Zu} 100 \mathrm{com}$ gelöst, drehen :

100 Teile Invertzucker . . . . . . $-21,5^{\circ}$

$$
\begin{aligned}
& 34,77 " \quad \text {. . . . . . }-21,5 \times 0,3477=-7,48^{\circ} \\
& 100 " \text { Extrakt aus Stärkesirup . . }+134,1^{\circ} \\
& 28,53 " \quad \Rightarrow \quad \text { " } \quad \text {. } \quad+134,1 \times 0,2853=+38,26^{\circ} \\
& 11,28, \text { Extrakt aus Pflaumen . . . . . . . . . } \pm 0 . \\
& 74,58 \text { Teile Extrakt aus Marmelade . . . . }+30,78^{\circ} \\
& 100 \\
& \mathrm{D}=+41,27^{\circ} \text {. }
\end{aligned}
$$

Dieser spezifischen Drehung entspricht in der Tabelle von Juckenack ein Gehalt des invertierten Extraktes an wasserhaltigem Stärkesirup von $49,22 \%$.

$100 \mathrm{~g}$ Extrakt enthalten . . . . . . . . $49,22 \mathrm{~g}$

$74,58 " \quad \% \quad$ oder $100 \mathrm{~g}$ Marmelade enthalten $49,22 \times 0,7408$ $=36,70 \%$ Stärkesirup.

Dieser Wert weicht von den tatsächlich vorhandenen $34,77 \%$ nur um $1,93 \%$ ab. Subtrahiert man hingegen von der spezifischen Drehung der Marmelade den 
Wert 21,5, so entspricht der Differenz $41,27-21,5=19,77^{\circ}$ nach der Jucken ackschen Tabelle ein Gebalt von 32,32\% und für die Marmelade ergibt sich ein Stärkesirupgehalt von $32,32 \times 0,7458=24,10 \%$. Dieser Wert ist aber um $10,67 \%$ zu niedrig!

Noch günstiger stellt sich die Sachlage für die Juckenack'sche Methode, wenn man berïcksichtigt, daß die spezifische Drehung des Zwetschenextraktes in der Regel nicht Null, sondern ziemlich stark negativ ist. Lediglich im Pflaumenmus, welches durch sehr starkes Einkochen der Früchte gewonnen wird, kann durch teilweise Zerstörung der Fructose eine Verminderung der Linksdrehung bis auf 0 verursacht werden. In den Marmeladen wird hingegen meist die unveränderte Drehung des Pflaumenextraktes mit ungefähr $-10^{\circ}$ zur Geltung gelangen. Es besteht daher kein Bedenken, die Juckenack'sche Methode auch für Pflaunenmarmelade in Anwendung zu bringen, um so mehr als die beobachteten Abweichungen durchaus innerhalb der vereinbarten Feblergrenzen liegen.

Aus vorstehenden Darlegungen sind folgende Schlüsse zu ziehen:

1. Es ist nicht richtig, bei der Bestimmung des Stärkesirupgehaltes in Pflaumenmus und Pflaumenmarmeladen von der ermittelten spezifischen Drehung des invertierten Extraktes den Wert 21,5 zu subtrahieren und dann die Juckenack'sche Tabelle zu benutzen.

2. Bei der Untersuchung von Pflaumenmus ohne Zusatz von Rohrzucker muß man vielmehr die Formel $\mathrm{x}=\frac{100}{134,1} \times \mathrm{D}$ anwenden, in welcher den prozentischen Gehalt des invertierten Extraktes an wasserfreiem Stärkesirup und D die spezifische Drehung des Extraktes darstellt.

3. Zur Bestimmung des Stärkesirupgehaltes in Pflaumenmarmeladen empfiehlt es sich, die Juckena ck'sche Methode unverändert beizubehalten.

Um die vorstehenden Deduktionen auf ihre Berechtigung prüfen zu können, habe ich eine hiesige angesehene Fabrik von Obstkonserven gebeten, in meinem Beisein einige Proben Pflaumenmarmelade herzustellen. Als Ausgangsmaterial für die mit größter Bereitwilligkeit angestellten Versuche diente ein Pflaumenmark, von dem $240 \mathrm{~kg}$ mit $160 \mathrm{~kg}$ Zucker zu $298 \mathrm{~kg}$ Marmelade eingekocht wurden. Die von Herrn Dr. Simmich ausgefübrte Analyse des Pflaumenmarks und der fertigen Marmelade ergab folgende Werte, neben welchen des Vergleichs halber die durch Berechnung aus den Einzelbestandteilen erhaltenen angeführt sein mögen:

\begin{tabular}{|c|c|c|c|}
\hline \multirow[b]{2}{*}{ Ausgeführte Bestimmangen } & \multirow[b]{2}{*}{$\begin{array}{l}\text { Pflaumen- } \\
\text { mark }\end{array}$} & \multicolumn{2}{|c|}{ Pflaumenmarmelade } \\
\hline & & $\begin{array}{l}\text { Analytisch } \\
\text { bestimmte } \\
\text { Werte }\end{array}$ & $\begin{array}{c}\text { Berechnete } \\
\text { Werte }\end{array}$ \\
\hline 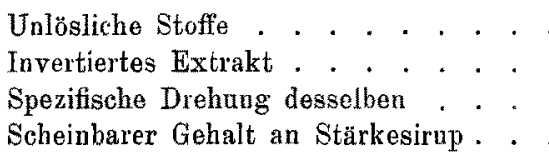 & $\begin{array}{l}1,70 \% \\
15,20 \\
\pm 0 \\
2,56 \%\end{array}$ & $\begin{array}{r}1,06 \% \\
64,90 \% \\
-13,670 \\
4,17 \%\end{array}$ & $\begin{array}{r}1,37 \% \\
68,75 \% \\
-15,110 \\
3,58 \%\end{array}$ \\
\hline
\end{tabular}

Hieraus ergibt sich, daß bei der Untersuchung dieser reinen Marmelade nach dem Verfahren von Juckenack ein scheinbarer Gehalt an Stärkesirup von 4,17\% gefunden wurde. Daß dieser Gehalt etwas größer ist als die rechnerisch ermittelte Zahl, erklärte sich aus dem Umstande, daß der Extrakt anscheinend durch Zersetzungen 
beim Kochen zurückgeht, und daß gleichzeitig die Linksdrehung der invertierten lösung wegen der größeren Empfindlichkeit der Fructose eine Verringerung erfährt. Die Abnahme der unlöslichen Stoffe dürfte mit der von uns regelmäßaig beobachteten Zunahme der löslichen Pektinstoffe zusammenhängen, welche einer liebenswürdigen privaten Mitteilung von Herm Professor Rosenthaler zufolge auf der Löslichkeit der Pektinstoffe in Zuckerlösungen beruht ${ }^{1}$ ).

Beim Vermischen der oben analysierten reinen Pflaumenmarmelade mit Stärkesi rup von mittlerer Zusammensetzung ergeben sich folgende Werte:

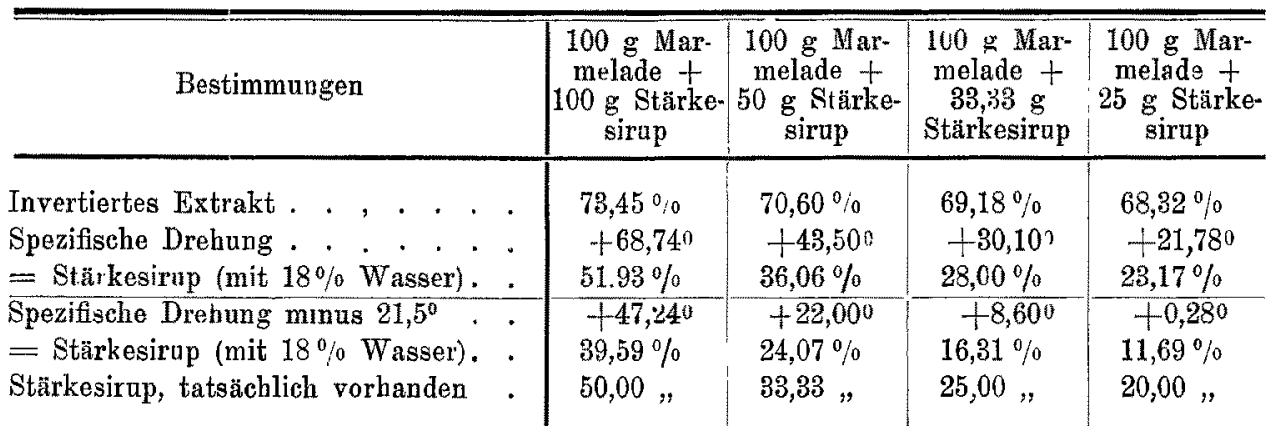

Hiernach liegt der durch Anwendung der Jucken a ck'schen Methode bedingte Fehler auch bei Pflaumenmarmelade innerhalb der vereinbarten Latitüde, während nach $\mathrm{Ab}$ zug von 21,5 von der spezifischen Drehung viel zu niedrige Werte erhalten werden.

\section{Nachtrag.}

Auf S. $168 \mathrm{ff}$. des laufenden Bandes dieser Zeitschrift lassen die Herren Härtel und Solling der von Simmich und mir veröffentlichten Abhandlung über die Untersuchung und Beurteilung von Marmeladen ${ }^{2}$ ) die Ehre einer Besprechung zuteil werden, welche mich zu einer kurzen Entgegnung zwingt. Denn wenn wir auch bei Anfügung des hier seit langen Jahren erprobten Analysenganges der Überzeugung Ausdruck verliehen haben, daß sich an demselben noch manche zweckmäßige Änderung und Verbesserung anbringen lassen wird, so glaube ich doch nicht, daß hierzu gerade die von IÏrtel und Sölling gemachten Vorschläge geeignet sind, da sie zwar Abänderungen aber nichts weniger als Verbesserungen darstellen.

Dab die Herstellung der Grundlösung durch Auffüllen der abgewogenen Marnelade zu einem bestimmten Volumen theoretisch nicht richtig ist, bedurfte keines Beweises; der durch das Volumen der unlöslichen Stoffe verursachte Fehler ist jedoch so gering, daß er im Vergleich zu den zahlreichen übrigen Fehlerquellen gar nicht in Betracht kommt. Will man ihn aber vermeiden, so braucht man nur nach dem Vorschlage von Juckenack die unlöslichen Stoffe auszuwaschen und das Filtrat aufzufüllen. Weshalb $\mathrm{Härtel}$ und Sölling dieser allgemein üblichen Arbeitsweise gegenüber zu dem überaus umständlichen und zeitraubenden Verfahren greifen, daß sie die wässerige Lösung nicht zu einem bestimmten Volumen, sondern zu einem bestimmten Gewichte, beispielsweise $1009,97 \mathrm{~g}$ oder $527,035 \mathrm{~g}$, auffüllen und dann für jede einzelne Bestimmung aliquote Teile abwägen, ist nicht recht

1) Vergl. Tschirch, Ber. d. Deutsch. Pharm. Ges. 1907, 17, 237.

$\left.{ }^{2}\right)$ Diese Zeitschrift 1910, 20, 241. 
ersichtlich, denn der angegebene Grund: Abneigung gegen das Abpipettieren von Zuckerlösung und mangelhafte Übereinstimmung der geeichten Meßgeräte hat bislang noch keinen Chemiker abgehalten, sich der Vorteile des Abmessens und der Mabanalyse überhaupt zu bedienen. Auch $\mathrm{Härtel}$ und Sölling nicht! Nach ihrer eigenen Angabe auf S. 172-173 wägen sie zwar für die Zuckerbestimmung $50 \mathrm{~g}$ der Grundlösung ab, füllen dann aber rubig zu einem bestimmten Volumen $(1000 \mathrm{ccm})$ auf (Meßkolben!) und verwenden nachher $25 \mathrm{ccm}$ (doch wohl mit der Pipette gemessen?) zur Behandlung mit Fehling'scher Lösung. Auch auf Seite 175 steht zu lesen, daß die abgewogene Menge Grundlösung in einem $200 \mathrm{cem}-\mathrm{Kolben}$ aufgefüllt wird, und so geht es durch die ganze Arbeit. Also überall Meßkolben und Pipetten, warum dann zu Anfang die umständliche Wägerei!

Bei der Zuckerbestimmung nehmen Härtel und Sölling Anstoß an der Verwendung der Tierkohle, die ja in der Tat manche Nachteile hat. Über Bleiessig und Tierkohle sind schon Bänrle gescbrieben, und es dürfte nur wenige Fachgenossen geben, denen über dieses Thema noch etwas Neues berichtet werden kann. Aber die Behandlung mit Tierkohle ist nun einmal ein integrierender Bestandteil der vereinbarten Juckenack'schen Methode, und ich möchte doch dringend warnen, schon jetzt an dieser Abänderungen zu treffen, deren Wert sich zurzeit noch nicht übersehen läßt. Was will der Einfluß der Tierkohle besagen gegenüber den bekannten Fehlern, welche die Übertragung der Invertzuckerbestimmung auf Gemische von Saccharose, Invertzucker, Glykose und Dextrin im Gefolge hat und welche nur durch Anwendung der Kjeldahl'schen Methode verringert werden könnten.

In bezug auf die Bestimmung der spezifischen Drehung des invertierten Extraktes stimmen Härtel und Sölling unserer Auffassung bei, daß diese Drehung auf die Menge des invertierten Extraktes bezogen werden muß, und das war gerade der springende Punkt unserer Arbeit. Sie ziehen es allerdings vor, die Menge des invertierten Extraktes statt aus dem spezifischen Gewichte der invertierten Lösung aus dem gesondert bestimmten Saccharosegehalt abzuleiten, und ich gebe gern zu, daß man auch auf diese Weise richtige Werte erlangen kaun, allerdings auf umständlicherem Wege als nach unserem Vorschlage, weil eine Polarisation oder Zuckerbestimmung mehr ausgeführt werden muß. Hingegen kann ich den gegen unser Verfahren erhobenen Einwand, daß man das spezifische Gewicht der Salzsäure jedesmal von neuem bestimmten müsse, nicht als stichhaltig anerkennen, vorausgesetzt, daß man die rauchende Salzsäure nicht gerade in einer offenen Porzellanschale aufbewahrt. Nach Versuchen von Si m m i ch sank das spezifische Gewicht einer in offenem Zylinder befindlichen Salzsäure während 14-tägiger Aufbewahrung von 1,1870 auf 1,1550; wenn man die Flasche hingegen geschlossen hielt und nur zum täglichen Gebrauche öffnete, so betrug das spezifische Gewicht nach 14 Tagen noch 1,1858, und das spezifische Gewicht der Verdünnung 5:100 ging dementsprechend in der gleichen Zeit von 1,0110 nur auf 1,0109 zurück. Wie Härtel und Sölling zu ihren Abweichungen von 5 Einheiten in der 4. Dezimale gekommen sind, ist aus ihrer Arbeit nicht recht ersichtlich. Möglicherweise haben sie mit verschiedenen Proben rauchender Salzsäure gearbeitet, die natürlich verschiedene Werte ergeben müssen. Bei Benutzung derselben Salzsäure genügt es, alle 14 Tage das spezifisch neu zu bestimmen.

Für die Berechnung des Stärkesirups schlagen Härtel und Sölling vor, den einen Grenzwert der Jucken ack'schen Tabelle $-21,5^{\circ}$ durch die Zahl 
- 20,420 zu ersetzen, welche sie auf Grund dreier Versuche als die richtige spezifische Drehung des Invertzuckers ansehen. Diese Auffassung stimmt mit den Ergebnissen unserer Bestimmungen (1. c. S. 255) und mit den Angaben der älteren Autoren nicht überein, vielmehr ergibt sich die spezifische Drehung des Invertzuckers bei $20^{\circ}$ sowohl nach den Arbeiten $\nabla$. Lippmann's als auch nach der Clerget.Formel zu $-21,5^{0}$. Die bekannten Einflüsse verschiedener Faktoren, wie der Konzentration und Reaktion der Lösung, sind noch zu wenig klargestellt, als daf sie die Abänderung dieser grundlegenden Zahl wünschenswert erscheinen ließen.

Wenn ich dann zum Schlusse noch die komplizierte, jeden Mathematiker mit Grauen erfüllende Formel auf S. 178 ins Auge fasse, die abgesehen von ihrer Unförmlichkeit noch mehrere Fehler enthält, so möchte ich bezweifeln, daß viele Fachgenossen sich den Vorschlägen $\mathrm{Härtel's} \mathrm{und} \mathrm{S}$ ölling's anschließen werden, sondern viel eher meinen, daß sie bei der altgewohnten Arbeitsweise, die wir lediglich in ein paar Sätze zusammengefaßt haben, verharren werden.

\title{
Zum Nachweis von Fuselöl in Trinkbranntwein.
}

\author{
Von \\ Eduard Herzog, k. k. Assistent. \\ Mitteilung aus der K.-k. Untersuchungsanstalt für Lebensmittel in \\ Czernowitz.
}

[Eingegangen am 21. Dezember 1910.]

Eine einfache und dabei zuverlässige Methode zum Nachweis von Fuselöl in Trinkbranntwein ist trotz der vielen in Vorschlag gebrachten Reaktionen bisher nicht bekannt geworden. In letzter Zeit wurden einige Farbenreaktionen zum Nachweise von Amylalkohol empfohlen, über welche in dieser Zeitschrift ${ }^{1}$ ) berichtet wurde.

Eine neue Farbenreaktion, welche angeblich in einfacher Weise den Nachweis von Amylalkohol ermöglicht, gab $\mathrm{Hugo} H o l l a ̈ n d e r^{2}$ ) bekannt; sein Verfahren ist folgendes:

„25 cem des zu prüfenden Branntweins werden mit $1 \mathrm{~cm}$ Normalkalilauge versetzt und in den Destillierkolben gebracht. Nach Abdampfung der ganzen Flüssigkeitsmenge wird zu eiwa $5 \mathrm{ccm}$ des Destillates ebensoviel konzentrierte Essigsäure in einem Reagensglas hinzugesetzt, die Mischung durch Erhitzen bis zum Sieden gebracht und etwa 1 Minute lang weitergekocht. Sodann wird dem Gemisch ein einziger Tropfen Phenylhydrazinum purum (M erck) beigegeben, die klare Lösung wieder aufgekocht und dann durch Abkühlung auf Eis oder in fließendem Wasser auf Normaltemperatur gebracht. Dasselbe kann auch durch ruhiges Stehenlassen bewirkt werden.

1) Vergl. Komarowsky, diese Zeitschrift 1904, 7, 568 und P. Tokahashi, di. se Zeitschrift 1906, 11, 353.

2) Münch, m:d. Wochenschr. 1910, 57, 82-88; Chem. Zentralbl. 1910, I, 671. 